\section{Simulation based resuscitation training as an alternative to the four stage approach; combining skills and human factors in one session}

Svetlana Nela - Senior Resuscitation Practitioner, BSc (Hons), Brighton, UK

Study purpose: The 'four stage approach' has been the traditional method used to train healthcare staff in resuscitation skills and has been implemented worldwide, as a standard model. However, despite that, evidence suggests this teaching method does not demonstrate better improvements when compared with other methods (Jenko et al 2012, Munster et al 2016). Additionally there is limited data on staff confidence post resuscitation training although evidence suggests a poor retention of resuscitation skills (Smith at al 2008).

Traditional 4 stage approach (Bullock et al 2018)

- Stage 1 Demonstration of the skill, performing at real speed with or without speech.

- Stage 2 Repeat demonstration with dialogue,

providing the rationale for actions.

- Stage 3 Repeat demonstration guided by one or more of the learners.

- Stage 4 Repeat demonstration by the learner, and practice of the skill by all learners

Ullock et al 2018 Pocket Guide for Teaching for Clinical Instructors. Carr (2006) cited in Bullock 2018.

. Munstel et al (2016) https://www.ncbi.nIm.nih.gov/pmc/articles/PMC5003127/
Smith et al (2008) https://www.ncbi.nlm.nih.gov/pubmed/18406037 McConaughey (2008) https://www.ncbi.nlm.nih.gov/pubmed/18496068

The four stage model focuses on performing a range of technical skills, resuscitation principles and implementation of guidelines. However it only briefly prepares individuals for how to adapt to the unexpected, changeable work environment of resuscitation event, when clinicians are expected to multitask and maintain situational awareness. This can lead to a reduction of chest compression quality, even in a simulated environment. The existing Crew Recourses Management (CRM) training in contrast does not focus on failure or correction in performing clinical skills and only focuses on team dynamics and behavior, McConaughey (2008).

SIMCAD
- Baseline simulation - 2 minute sim where the team
manages a collapsed patient without instructor
comments
- Instructor facilitated learning conversation using
(Carr 2006) de-brief, discussing key issues and skills
gaps and encouraging students to teach each other
with instructor guidance.
- Series of simulation scenarios with de-brief as
learning conversation, using clear role allocation
and leadership to build up individual resuscitation
skills and improve team dynamics
- Final simulation with de-brief and reflection on
improvement
- Post course, we recommend a daily emergency
briefing and role allocation to enable efficient skill
distribution so the individual and team can adapt to
daily variables

Method: We developed a short curriculum which combines proficiency in technical and none technical skills (TEAM approach) adopted an alternative resuscitation training model for our in-house SimCAD (Simulated CPR and Defibrillation) course. It is based on assessing existing team knowledge but identifying any individual skill gaps. This allows instructor and the candidate to build up skills based on both team and individual learning needs. It also creates a safe environment for each candidate to identify their personal strengths and weaknesses and explore different coping mechanisms in emergency situations whilst efficiently using existing resources. We recommend a ratio of 1 instructor per 6 candidates. The course was evaluated with structured questionnaires.
Trial: The initial trial was carried out in an Acute Trust with 986 acute beds and formed part of mandatory training for medical and nursing staff. Data was collected for a period of 8 weeks and immediately after each SimCAD. 65 nurses and doctors participated in the evaluation.

Results and Conclusion: The results demonstrated that candidates valued the initial simulation, de-brief and team approach during these session. Participants felt not only more confident dealing with resuscitation scenarios, but highlighted an improvement in efficiency and task prioritisation. Combining technical and non technical skills in one session can be an alternative to a traditional four stage method in delivering mandatory resuscitation training.

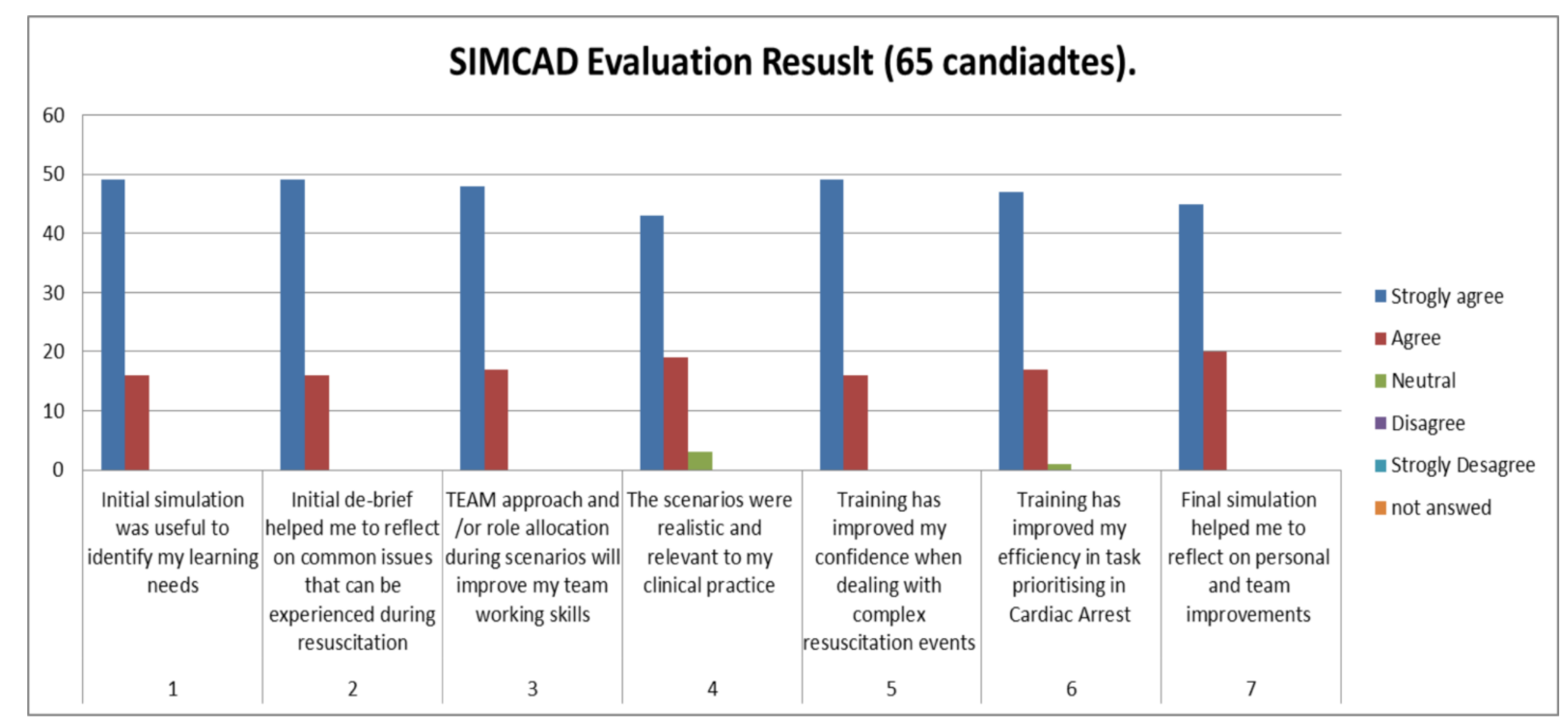

